\title{
Reversible Acute Parkinsonism and Bilateral Basal Ganglia Lesions in a Diabetic Uremic Patient
}

\author{
Hipólito Nzwalo ${ }^{a}$ Francisca Sá $^{a}$ Carlos Capela $^{b}$ \\ Fátima Ferreira $^{a}$ Carlos Basílio $^{a}$ \\ ${ }^{a}$ Neurology Department, Faro Hospital EPE, Faro, and ${ }^{b}$ Neurology Department, \\ Hospital dos Capuchos, Lisbon, Portugal
}

\section{Key Words}

Acute parkinsonism $\cdot$ Uremia $\cdot$ Basal ganglia

\section{Abstract}

The syndrome of bilateral basal ganglia lesions in diabetic uremic patients is a rare disorder mostly reported in Asians. There are few reports of the syndrome in Caucasians. It manifests as an acute hyperkinetic or hypokinetic extrapyramidal disorder in association with uniform neuroimaging findings of bilateral symmetrical basal ganglia changes in diabetics undergoing hemodialysis. Its pathophysiology remains largely unknown. Thus, we report a typical case of the syndrome in a Caucasian patient who developed an acute and reversible akinetic rigid parkinsonism secondary to bilateral basal ganglia lesions.

\section{Introduction}

The syndrome of bilateral basal ganglia lesions in diabetic uremic patients is a rare disorder mostly reported in Asians [1-3]. It manifests as an acute hyperkinetic or hypokinetic extrapyramidal disorder in association with uniform neuroimaging features of bilateral symmetrical basal ganglia changes in diabetics undergoing hemodialysis [1-3].

\section{Case Report}

A 62-year-old man, with arterial hypertension, hypercholesterolemia, and type 2 diabetes mellitus causing end-stage renal disease (ESRD) on twice weekly hemodialysis for the last 3 years, presented with a 2-week history of gradual-onset slurred speech, gait disturbance and decreased spontaneity. 
There was no recent history of hypoglycemia, hyponatremia, hypoxia, monoxide carbon intoxication, hypotension or other metabolic or electrolyte disturbance. On general examination, he was stable with normal cardiopulmonary sounds and no peripheral edema. Neurological examination disclosed hypophonic dysarthria, bradykinesia, cogwheel muscle rigidity, postural instability, and gait disturbance. There was no resting tremor, asterixis, or myoclonus. Blood urea nitrogen $(60 \mathrm{mg} / \mathrm{dl})$ and serum creatinine levels $(3.3 \mathrm{mg} / \mathrm{dl})$ were within the normal range. Blood glucose level was 128 $\mathrm{mg} / \mathrm{dl}$ and glycated hemoglobin was $6.8 \%$. Gasometry was normal, and levels of manganese, copper, and lead were within the normal range as well. Brain MRI showed bilateral and symmetrical lesions of the basal ganglia, with low-signal intensity on T1-weighted images, and increased signal intensities on T2-weighted and fluid attenuation inversion recovery (FLAIR) imaging (fig. 1a-f). The cytochemical analysis of the cerebrospinal fluid was normal. He started ropinirole initially - $1 \mathrm{mg}$ three times daily, and subsequently, levodopa-carbidopa - 100/25 mg three times daily. Dialysis was intensified to 3 times weekly. His clinical condition improved after the second week, and at the end of third week he was asymptomatic. Brain MRI showed almost complete resolution of the lesions (fig. $1 \mathrm{~g}, \mathrm{~h}$ ). The antiparkinsonian treatment was interrupted. He resumed his normal daily activities, and after 1 year of follow-up remains without any symptoms or signs of neurological disease.

\section{Discussion}

Our patient presented with the typical clinical and imaging findings of the syndrome of bilateral basal ganglia lesions in diabetic uremic patients. This syndrome was first described by Wang et al. [1], in 1998, and is characterized by acute hyperkinetic and/or hypokinetic extrapyramidal movement disorders in association with uniform neuroimaging findings of bilateral symmetrical basal ganglia changes in diabetic ESRD patients undergoing hemodialysis [2,3]. The imaging findings are a hallmark of this syndrome. Brain CT shows decreased density in the basal ganglia bilaterally, and brain MRI usually shows decreased signal intensity on T1-weighted images and increased signal intensity on T2-weighted images over the same areas. In some cases, perifocal edema and mass effect can be seen. These lesions often regress or disappear during follow-up, but irreversible cystic degeneration in the medial areas of the basal ganglia can occur [1-3]. There are fewer than 20 cases of this syndrome, mostly from Asia, and only 3 reports in Caucasians [4-6]. The pathophysiology remains largely unknown. Factors such as uremic toxins, diabetic microangiopathy, metabolic acidosis, and hypoglycemia may be implicated [1-6]. Apart from symptomatic treatment, dialysis intensification is indicated under the assumption of a possible underlying toxic or metabolic mechanism related to the diabetic renal failure [2, 5]. 

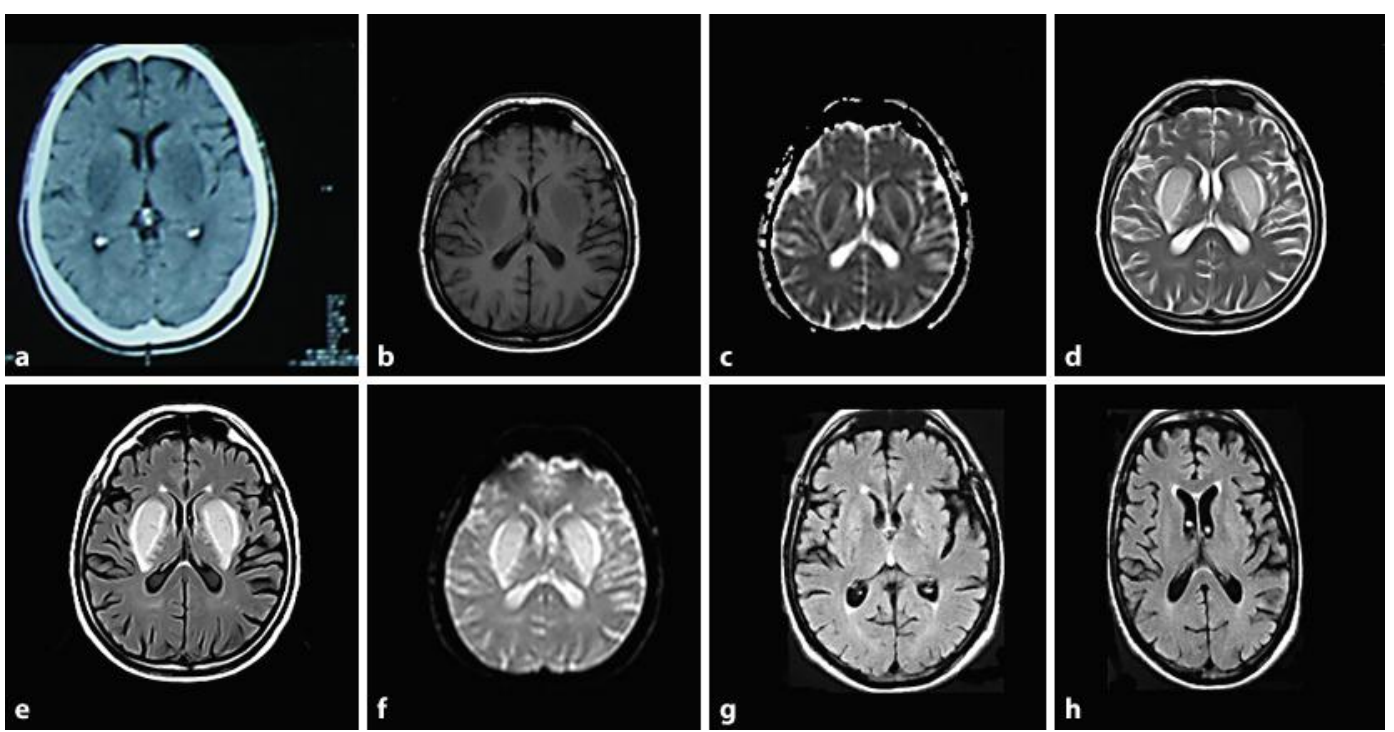

Fig. 1. Bilateral basal ganglia lesions at presentation (a-e): hypodense on CT (a); hypointense on T1weighted image (b) and apparent diffusion coefficient map (c); hyperintense on T2-weighted (d), FLAIR (e) and diffusion-weighted images (f). Residual lesions after 3 weeks: T1-weighted (g) and FLAIR images (h).

\section{References}

1 Wang HC, Brown P, Lees AJ: Acute movement disorders with bilateral basal ganglia lesions in uremia. Mov Disord 1998;13:952-957.

-2 Wang HC, Cheng SJ: The syndrome of acute bilateral basal ganglia lesions in diabetic uremic patients. J Neurol 2003;250:948-955.

-3 Lee EJ, Park JH, Ihn Y, Kim YJ, Lee SK, Park CS: Acute bilateral basal ganglia lesions in diabetic uremia: diffusion-weighted MRI. Neuroradiology 2007;49:1009-1013.

4 Cupidi C, Piccoli F, La Bella V: Acute reversible parkinsonism in a diabetic-uremic patient. Clin Neurology Neurosurgery 2006;108:601-603.

5 Li JY, Yong TY, Sebben R, Khoo E, Disney AP: Bilateral basal ganglia lesions in patients with end-stage diabetic nephropathy. Nephrology (Carlton) 2008;13:68-72.

-6 Juryńczyk M, Rozniecki J, Zaleski K, Selmaj K: Hypoglycemia as a trigger for the syndrome of acute bilateral basal ganglia lesions in uremia. J Neurol Sci 2010;297:74-75. 